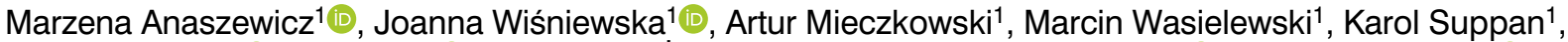
Beata Czerniak ${ }^{1}$ (D), Kinga Lis $^{2}$ (D), Magdalena Żbikowska-Gotz², Zbigniew Bartuzi² ${ }^{2}$, Jacek Budzyński ${ }^{1}$ (D)

${ }^{1}$ Department of Vascular and Internal Diseases, Faculty of Health Sciences, Ludwik Rydygier Collegium Medicum in Bydgoszcz, Nicolaus Copernicus University in Torun, Bydgoszcz, Poland

2Department of Allergology, Clinical Immunology and Internal Diseases, Faculty of Medicine, Ludwik Rydygier Collegium Medicum

in Bydgoszcz, Nicolaus Copernicus University in Torun, Bydgoszcz, Poland

\title{
Soluble vascular cell adhesion molecule-1 in patients with non-valvular atrial fibrillation
}

\section{Corresponding author:}

Jacek Budzyński, Department of Vascular and Internal Diseases, Faculty of Health Sciences, Ludwik Rydygier Collegium Medicum in Bydgoszcz, Nicolaus Copernicus University in Torun, Bydgoszcz, Poland e-mail: budz@cps.pl
Medical Research Journal 2020; Volume 5, Number 3, 158-166 10.5603/MRJ.a2020.0032 Copyright (C) 2020 Via Medica ISSN 2451-2591

\begin{abstract}
Background: Disturbances in atrial microcirculation is recognized as a risk factor for atrial fibrillation (AF). AIM: The aim of this study was to determine the associations between circulating soluble vascular cell adhesion molecule-1 (sVCAM-1) and the risk of AF and a one-year prognosis among consecutive inpatients. Methods: Eighty consecutive inpatients hospitalized due to non-valvular AF and 80 consecutive inpatients admitted for exacerbation of chronic coronary syndrome (control group) were enrolled in the study. A cardiologic workup was performed and blood SVCAM-1 concentration was determined using the ELISA method. Results: Patients with AF had similar blood sVCAM-1 concentration compared to the control group. AF patients treated with new oral anticoagulants (NOACs) were significantly less likely to have a sVCAM-1 concentration elevated above the median value than patients treated with warfarin $(34.2 \%$ vs $65.8 \%$; $p$ $=0.01)$. Patients with an increased percentage of fat mass (FM) had lower sVCAM-1 concentration. The risk of all-cause mortality and MACE during follow-up rose in individuals with elevated sVCAM-1 $(\geq 1242$ and $\geq 587 \mathrm{ng} / \mathrm{ml}$, respectively) with (OR; 95\%Cl): 5.39; 1.57-18.45; $p=0.007$, and 6.00; 1.18-30.37; $p=0.03$, respectively. Risk of death rose with increase in the ratio of sVCAM-1 and FM (1.02; $1.00-1.04 ; p=0.019)$. Conclusions: Elevated SVCAM-1 was associated with all-cause mortality and MACE during one-year follow-up, but do not links the risk of AF. Use of NOACs may favorable affect endothelial function, A lower level of sVCAM-1 in obese patients may mediate the phenomenon of the "obesity paradox" in patients with AF. Key words: sVCAM-1; atrial fibrillation; thromboembolic complications; mortality, obesity paradox; new oral anticoagulants
\end{abstract}

Med Res J 2020; 5 (3): 158-166

\section{Introduction}

Atrial fibrillation (AF) is one of the most prevalent forms of cardiac arrhythmia [1]. AF can occur in patients with cardiovascular disorders, such as coronary artery disease, hypertension and valvular heart disease, and those with extra-cardiac disturbances, thyrotoxicosis and dyselectrolytemia, for example. These disturbances can affect many pathomechanisms, leading to AF. One of the AF substrates is local heterogeneity of refractions in atrial muscle cells, cardiac pacemakers and the conduction system, which may be caused by ischemia. In turn, myocardial ischemia may result from an imbalance in the blood supply through coronary arteries and/or oxygen demand
[1]. One potential cause of this type of disturbance is endothelial dysfunction [2].

Endothelial dysfunction is a risk factor for several cardiovascular disorders $[3,4]$. This type of disturbance reduces the reserve of vascular dilatation, facilitates arterial thrombosis and promotes migration and proliferation of vascular wall cells $[3,4]$. The most prevalent cause of endothelial dysfunction is an inadequate or delayed modification of atherosclerosis risk factors (e.g. smoking, hypertension, diabetes and hyperlipidemia). There are many markers of global endothelial dysfunction. These markers can be invasive, such as intra-arterial application of acetylcholine and/or nitroglycerin during coronarography, or non-invasive, such as e.g. brachial artery flow-mediated dilatation or tonometry of peripheral 
arteries (digital pulse amplitude tonometry [PAT]). Of the biochemical markers of endothelial dysfunction, the one most prevalently used is blood concentration of endothelin-1 and adhesion molecules, e.g. E-selectin, P-selectin, intercellular adhesion molecule-1 (ICAM-1), and soluble vascular cell adhesion molecule-1 (sVCAM-1) [3]. Soluble VCAM-1 is also recognized as a biomarker of inflammation [5] and endothelial activation [2].

The role of endothelial dysfunction in patients with atrial fibrillation remains understudied [3-7]. However, it has been reported that the combined dysfunction of cardiac (i.e. endocardial, microvascular and coronary) and vascular endothelial cells may affect atrial and ventricular cardiomyocyte contractility and remodelling and lead to fibrosis. Endothelial dysfunction may also lead to reduced synthesis of substances with antithrombotic, anti-inflammatory and anti-proliferative properties, such as tissue plasminogen activator and nitric oxide (due, i.a., to an increased level of the amino acid asymmetric dimethylarginine [ADMA], an endogenous inhibitor of NO synthase [eNOS]) [7], as well as to increased secretion of procoagulant factors, e.g. von Willebrand factor (VWF) [7], overexpression of adhesion molecules (e.g. sVCAM, sICAM, P-selectin and E-selectin) [5], and increase of growth factors (e.g. fibroblast growth factor [bFGF] and vascular endothelial growth factor [VEGF]) [3]. Through the pathomechanisms mentioned, endocardial and vascular endothelial dysfunction may take part in the pathogenesis of AF occurrence, recurrence of arrhythmia, the formation of permanent forms of AF, and risk of thromboembolic complications [3-8].

On the basis of the premises mentioned above, we formulated the hypothesis that blood SVCAM-1 concentration could, as a biomarker of altered systemic endothelial function, indicate the risk of heart atria ischemia and cardiac arrhythmia induced by the dispersion of the refraction time [3]. As endothelial function and angiogenesis are both related to AF risk and body mass and composition [9], we assumed that endothelial dysfunction may operate as a link between the risk of AF and obesity $[10,11]$. The aim of this study was to determine the associations between circulating sVCAM-1 and the risk of AF, type of arrhythmia, risk of thromboembolic and hemorrhagic complications and one-year prognosis among consecutive inpatients. The other purpose of this study was to estimate the role of sVCAM-1 as a link between the risk of AF and obesity.

\section{Patients and methods}

\section{Patients}

This cohort study was performed with 80 consecutive patients hospitalized due to non- valvular AF and a control group containing 80 consecutive inpatients hospitalized as a result of exacerbation of chronic coronary syndrome. All the study participants were admitted to the same clinic in an urban university hospital. The exclusion criteria were as follows: valvular heart disease; history or clinical signs of inflammatory processes or neoplasm; and lack of informed consent for participation in the study. Patients were recruited to the study between July 1, 2015, and December 31, 2016.

Medical history was obtained during the first day of hospitalization. A physical examination, laboratory tests, ECG and transthoracic echocardiography were also performed for every patient. After one year, a follow-up telephone interview was conducted with the patient or his or her relatives. Charlson Comorbidity Index (CCl) was scored in every patient.

\section{Parameters of nutritional status assessment}

Due to known associations between VCAM-1 and obesity, a nutritional status assessment was performed for all 160 study participants. The following parameters were measured: height $(\mathrm{cm})$, body weight $(\mathrm{kg})$, waist circumference (WC, cm), and hip circumference $(\mathrm{HC}, \mathrm{cm})$. Body composition was determined using bioelectrical impedance analysis (BIA) and a TANITA BC 420 MA device (TANITA Corporation, Japan). Each patient underwent a BIA examination between 7 am and $8 \mathrm{am}$, on an empty stomach and after urinating. The following BIA parameters were analyzed: FM (\%, kg), VAT score (in the range 1-59), fat-free mass (FFM, kg), and skeletal muscle mass (SMM, kg).

The following secondary parameters were calculated based on the above-mentioned indices:

- body mass index (BMI) $\left(\mathrm{kg} / \mathrm{m}^{2}\right)$ : calculated as the quotient of body mass expressed in $\mathrm{kg}$ and squared height expressed in $\mathrm{m}$;

- waist-to-hip ratio (WHR): calculated as the quotient of waist and hip circumferences (cm); the cut-off value for the diagnosis of the abdominal distribution of adipose tissue was 0.8 for females and 1.0 for males;

- waist-to-height ratio (WHtR): calculated as $100 x$ the quotient of waist circumference $(\mathrm{cm})$ and height $(\mathrm{cm})$;

- Body Shape Index $(\mathrm{ABSI})=\mathrm{WC} /\left[\left(\mathrm{BMI}^{2 / 3}\right)\right.$ $x$ (height ${ }^{1 / 2}$ )]

- Body adiposity index $(\mathrm{BAl})=\left(\mathrm{HC} /\right.$ height $\left.^{1,5}\right)-18$

\section{Biochemical and sVCAM-1 determinations}

Blood samples were taken from the ulnar vein of each patient between 7 am and 8 am on the day of admission while he or she was in a fasting state. Routine determinations were performed in an accredited laboratory at the university hospital and blood samples were taken to measure sVCAM-1 were centrifuged at 
$4^{\circ} \mathrm{C}$ and $3600 \mathrm{rev} / \mathrm{min}$. The serum obtained was stored at $-80{ }^{\circ} \mathrm{C}$ until required. sVCAM-1 (CD106) determination was performed using a commercially available enzyme-linked immunosorbent assay (ELISA) kit from the Biotech group (cat. no. 850.580.192) in accordance with the manufacturer's instructions.

Blood sVCAM-1 concentration was analyzed as a crude value and as a ratio to the following: blood C-reactive protein (CRP) concentration, fat mass (FM), and visceral adipose tissue (VAT). The rationale for such indexation of SVCAM-1 was that its secretion and serum concentration are related to the presence of inflammation (CRP, inflammation related to obesity and fat mass) [12], obesity and body composition (FM, VAT) [9, 13-19]. In this way, we can compare SVCAM-1 level adjusted to interpersonal diversity in inflammatory and adiposity status.

\section{Parameters of transthoracic echocardiography}

Transthoracic echocardiography was performed on admission for all the study participants by the same, experienced cardiologist using an Aplio transthoracic ultrasound device (TOSHIBA, Canon Medical Systems, USA) and a 2-5 $\mathrm{MHz}$ radial probe. The following echocardiographic parameters were determined: interventricular septum thickness (IVST) at end-diastole; posterior wall thickness (PWT); left ventricular end-diastolic dimension (LVEDD); maximal wall thickness (MWT), relative wall thickness (RWT); left ventricular ejection fraction (LVEF); left ventricular mass (LVM); and left atrium diameter indexed to body surface area (LAD/ BSA), left atrium area indexed to BSA (LAA/ BSA) and left atrium volume in apical four-chamber view indexed to BSA (LAV/ BSA). LVEF was assessed using the biplane, two-dimensional method of disks (modified Simpson's rule) in apical four- and two-chamber views. Left ventricular mass was calculated using the following formula: LVM $(\mathrm{g})=0.8 \times\left\{1.04 \times\left[\left([\text { LVEDD }+ \text { IVST }+ \text { PWT }]^{3}\right.\right.\right.$ - LVEDD $\left.\left.\left.{ }^{3}\right)\right]\right\}+0.6$. Body surface area was calculated using the following formula:

BSA $\left(\mathrm{m}^{2}\right)=0.01666667 \times$ height $0.5 \times$ body mass 0.5

\section{Measured outcomes}

For at least one year following discharge (median \pm interquartile range; 406; 374-442 days), the following outcomes were assessed measured by the same physician for each patient in the AF and control groups during a telephone interview with the patient or his or her relatives: all-cause mortality, all-cause readmission (e.g. due to acute coronary syndrome, cardiac heart failure exacerbation, arrhythmia recurrence) and stroke occurrence. The occurrence of composite end-point (major adverse cardiac event, MACE) was also analyzed and defined as the occurrence of any outcome measured (i.e. death or all-cause readmission or stroke).
The secondary outcomes comprised the above-determined parameters obtained during hospitalization both for patients with AF and those in the control group.

\section{Bioethics}

The investigation was conducted in compliance with the Declaration of Helsinki for medical research, after receiving permission from local Bioethical Committee No. 389/2015. Each patient gave written consent to participate in the study.

\section{Statistics}

Statistical analysis was conducted using a licensed version of statistical software STATISTICA version 13.1 (a data analysis software system) developed by StatSoft, Inc. (2017). The statistical significance level was set at a $p$-value $<0.05$. The normal distribution of the study variables was checked using the Shapiro-Wilk test. The results were mainly presented as the mean \pm standard deviation, or $\mathrm{n}, \%$. The statistical significance of differences between groups was verified using the Student's t-test, the Mann-Whitney U test and the Chi ${ }^{2}$ test. Spearman's correlations were checked to determine the relationships between SVCAM-1 and the clinical, biochemical and echocardiographic parameters. Survival analysis was performed by plotting the Kaplan-Meier curves for two probes and $\mathrm{F}$ Cox test for determination of statistical significance between them. Cut-off values for crude and indexed values of sVCAM-1 that have a predictive value for the risk of AF and the measured outcomes were determined for maximal Youden's indices by plotting the receiver operator curves (ROC). All the patients were then classified into two groups based on those cut-off values. For the qualitative variables obtained, the odds ratio (OR) was determined (using the free internet calculator available at https://www.medcalc.org/calc/ /odds_ratio.php) as the risk that AF would occur with an associated higher value of SVCAM-1, compared to the chance of AF occurring in patients with a lower value. The relationships between blood SVCAM-1 concentration and risk of $\mathrm{AF}$ and the measured outcomes were also verified using the logistic regression method.

\section{Results \\ Clinical characteristics \\ Compared to individuals in the control group, patients with AF were less prevalently smokers, were less likely to have a history of myocardial infarction, and tended to have a lower score on the $\mathrm{CCl}$ and HAS-BLED, as well as lower percentage of SMM. Moreover, they were more likely to have a higher}


Table 1. Comparison of clinical characteristics between the two groups studied

\begin{tabular}{|c|c|c|c|}
\hline Parameter & $\begin{array}{l}\text { Patients with atrial } \\
\text { fibrillation } \\
(\mathbf{n}=\mathbf{8 0})\end{array}$ & $\begin{array}{l}\text { Control group } \\
\quad(n=80)\end{array}$ & $\mathbf{p}$ \\
\hline Age (years) & $69.62 \pm 8.90$ & $69.18 \pm 8.44$ & 0.746 \\
\hline Male gender (n, \%) & $41(51.25 \%)$ & $42(52.50 \%)$ & 0.940 \\
\hline Smoking habit (n, \%) & 39 (48.75\%) & $65(81.25 \%)$ & $<0.001$ \\
\hline Hypertension (n, \%) & $68(85.00 \%)$ & $65(81.25 \%)$ & 0.636 \\
\hline Diabetes mellitus (n, \%) & $29(36.25 \%)$ & $34(42.50 \%)$ & 0.385 \\
\hline HAS-BLED score & $1.2 \pm 0.89$ & $1.9 \pm 0.95$ & $<0.001$ \\
\hline $\mathrm{CHA}_{2} \mathrm{DS}_{2}$ VASc score & $3.35 \pm 1.54$ & - & \\
\hline Past myocardial infarction (n, \%) & 9 (11.25\%) & $20(25.00 \%)$ & 0.022 \\
\hline CABG (n, \%) & $3(3.75 \%)$ & $9(11.25 \%)$ & 0.069 \\
\hline $\mathrm{CCl}$ score & $4.09 \pm 1.76$ & $4.94 \pm 1.96$ & 0.004 \\
\hline BMI $\left(\mathrm{kg} / \mathrm{m}^{2}\right)$ & $31.27 \pm 6.08$ & $27.04 \pm 4.77$ & $<0.001$ \\
\hline WHR & $0.99 \pm 0.09$ & $0.98 \pm 0.09$ & 0.677 \\
\hline WHtR & $0.68 \pm 0.47$ & $0.43 \pm 0.50$ & 0.001 \\
\hline ABSI & $0.085 \pm 0.005$ & $0.087 \pm 0.006$ & 0.038 \\
\hline BAl & $32.74 \pm 7.38$ & $29.84 \pm 6.11$ & 0.008 \\
\hline MNA score & $25.10 \pm 2.95$ & $23.89 \pm 3.18$ & 0.014 \\
\hline FM in BIA (\%) & $35.87 \pm 9.49$ & $29.91 \pm 9.08$ & $<0.001$ \\
\hline $\begin{array}{l}\text { Obesity diagnosis ( } 25 \% \text { FM for males; 35\% FM for } \\
\text { females) }\end{array}$ & $53(66.25 \%)$ & $35(43.75 \%)$ & 0.007 \\
\hline $\mathrm{FM}(\mathrm{kg})$ & $32.29 \pm 12.30$ & $22.63 \pm 9.42$ & $<0.001$ \\
\hline VAT score & $15.46 \pm 4.89$ & $12.10 \pm 3.87$ & $<0.001$ \\
\hline \multicolumn{4}{|l|}{ Measured outcomes during $420.82 \pm 56.58$ days of follow-up } \\
\hline Death $(\mathrm{n}, \%)$ & 8 (10.00\%) & $5(6.25 \%)$ & 0.402 \\
\hline Stroke $(n, \%)$ & $2(2.5 \%)$ & $2(2.5 \%)$ & 0.978 \\
\hline Myocardial infarction (n, \%) & 0 & 2 & 0.160 \\
\hline Readmission (n, \%) & 35 (43.75\%) & 47 (58.75\%) & 0.087 \\
\hline MACE (n, \%) & $44(55.00 \%)$ & $51(63.75 \%)$ & 0.219 \\
\hline
\end{tabular}

$\mathrm{ABSI}$ - A body shape index; BAI — body adiposity index; BIA — bioelectrical impedance analysis; BMI — body mass index; CABG — coronary artery bypass graft; $\mathrm{CCl}$ - Charlson Comorbidity Index; $\mathrm{CHA}_{2} \mathrm{DS}_{2} \mathrm{VASc}$ - evaluates stroke risk in patients with AF; FM - fat mass (expressed as $\mathrm{kg}$, and a \% of the whole body mass); FFM - fat-free mass; HAS-BLED - a scoring system for estimating bleeding risk in patients with AF; MACE - major adverse cardiovascular event combined with death, stroke, and all-cause rehospitalization; MNA — minimal nutritional assessment; VAT — visceral adipose tissue; WHR, waist-to-hip ratio; WHtR — waist-to-height ratio

MNA score, higher BMI, a higher percentage of fat mass and greater indices of visceral fat distribution (WHtR, ABSI, BAI, VAT score) (Tab. 1). Patients with $A F$ were also more likely to have lower values of LDL and higher blood concentrations of $\mathrm{NH}(2)$-terminal pro-brain natriuretic peptide (NT-proBNP) and hemoglobin (Tab. 2). The heart chambers of patients with AF also had thicker heart walls (IVST, PWT, MWT), larger dimensions of heart chambers (LVEDD, LAD/BSA, LAA/BSA, LAV/BSA) and a lower LVEF (Tab. 2).

\section{sVCAM-1 and parameters measured in patients with $\mathrm{AF}$}

Patients with AF did not differ from the control group in relation to serum SVCAM-1 concentration, although the SVCAM-1-to-CRP ratio was lower in patients with AF than in the control group (Tab. 2). Subgroups of patients with AF divided in relation to the type of arrhythmia experienced did not differ in relation to blood SVCAM-1 concentration either. The respective values of SVCAM-1 for patients with paroxysmal, persistent and permanent AF amounted to: 
Table 2. sVCAM-1, biochemical determinations and echocardiographic parameters for the two groups studied

\begin{tabular}{|c|c|c|c|}
\hline Parameter & $\begin{array}{l}\text { Patients with } \\
\text { atrial fibrillation } \\
\quad(n=80)\end{array}$ & $\begin{array}{l}\text { Control group } \\
(n=80)\end{array}$ & $\mathbf{p}$ \\
\hline sVCAM-1 (ng/ml) & $1264.14 \pm 620.95$ & $1241.18 \pm 593.56$ & 0.812 \\
\hline sVCAM-1/CRP (ng/mg) & $613.32 \pm 677.39$ & $964.52 \pm 1301.25$ & 0.035 \\
\hline sVCAM-1/FM (ng/ml/\%) & $39.12 \pm 25.66$ & $45.50 \pm 26.10$ & 0.117 \\
\hline sVCAM-1/VAT (ng/ml/score) & $94.15 \pm 64.94$ & $113.54 \pm 68.60$ & 0.070 \\
\hline Hemoglobin (g/L) & $136.00 \pm 14.70$ & $130.90 \pm 16.40$ & 0.039 \\
\hline LDL cholesterol (mg/dl) & $88.28 \pm 37.27$ & $101.29 \pm 41.24$ & 0.039 \\
\hline Triglycerides (mg/dl) & $110.70 \pm 49.80$ & $122.78 \pm 78.30$ & 0.249 \\
\hline Creatinine (mg/dl) & $1.04 \pm 0.35$ & $0.98 \pm 0.48$ & 0.401 \\
\hline CRP (mg/dl) & $12.94 \pm 30.45$ & $7.91 \pm 13.82$ & 0.183 \\
\hline NT-proBNP (ng/ml) & $2107.88 \pm 3040.70$ & $944.51 \pm 1854.53$ & 0.004 \\
\hline Interventricular septum (mm) & $12.02 \pm 1.83$ & $11.25 \pm 1.84$ & 0.009 \\
\hline Posterior wall thickness (mm) & $11.81 \pm 1.72$ & $10.82 \pm 1.92$ & 0.001 \\
\hline Maximal wall thickness (mm) & $11.92 \pm 1.74$ & $11.03 \pm 1.83$ & 0.002 \\
\hline Relative wall thickness (mm) & $0.48 \pm 0.11$ & $0.52 \pm 0.48$ & 0.497 \\
\hline Left ventricular mass /BSA $\left(\mathrm{g} / \mathrm{m}^{2}\right)$ & $120.71 \pm 34.38$ & $107.29 \pm 32.24$ & $<0.001$ \\
\hline $\operatorname{LVEF}(\%)$ & $53.12 \pm 10.14$ & $58.68 \pm 12.01$ & 0.002 \\
\hline LVEDD (mm) & $50.73 \pm 7.35$ & $47.75 \pm 8.40$ & 0.019 \\
\hline $\begin{array}{l}\text { Left atrium volume in apical four-chamber view indexed to BSA (ml/ } \\
\left.\mathrm{m}^{2}\right)\end{array}$ & $53.07 \pm 19.34$ & $38.68 \pm 12.36$ & $<0.001$ \\
\hline Left atrium diameter indexed to $\mathrm{BSA}\left(\mathrm{mm} / \mathrm{m}^{2}\right)$ & $23.07 \pm 4.88$ & $21.87 \pm 3.09$ & 0.068 \\
\hline Left atrium area in apical four-chamber view indexed to $\mathrm{BSA}\left(\mathrm{cm}^{2} / \mathrm{m}^{2}\right)$ & $13.43 \pm 3.10$ & $10.91 \pm 2.20$ & $<0.001$ \\
\hline
\end{tabular}

BSA — body surface area $\left(\mathrm{m}^{2}\right)$; CRP — C-reactive protein; FM — fat mass (expressed as a \% of whole body mass); LDL — low-density lipoprotein; LVEDD - left ventricular end-diastolic dimension; LVEF — left ventricular ejection fraction; NT-proBNP - NH(2)-terminal pro-brain natriuretic peptide; SMI — skeletal muscle mass index; sVCAM-1 — soluble vascular cell adhesion molecule-1; VAT — visceral adipose tissue. Conversion factors to SI units are as follows: for LDL cholesterol -0.0259 [mmol/l]; triglycerides -0.0113 [mmol/l]; creatinine -88.4 [mcmol/l]

$1238.73 \pm 620.61 \mathrm{ng} / \mathrm{ml} ; 1219.64 \pm 527.11 \mathrm{ng} / \mathrm{ml}$ and $1396.23 \pm 816.53 \mathrm{ng} / \mathrm{ml}$.

The associations between blood SVCAM-1 concentration and clinical factors were analyzed both in split analysis in subgroups of AF patients divided in relation to the median blood SVCAM-1 concentration ( $<$ and $\geq 1139 \mathrm{ng} / \mathrm{ml}$ ) and using non-parametric Spearman's correlations.

Patients with AF and blood sVCAM-1 concentration above the median value $(n=40)$ in comparison with patients with a blood sVCAM-1 concentration below the median value $(n=40)$ :

- were treated significantly less often with new direct oral anticoagulants (NOACs: $37.50 \%$ vs $62.50 \%$; $p=0.01)$, and patients treated with NOACs ( $n=38$, $47.50 \%$ ) were significantly less likely to have elevated blood sVCAM-1 concentration $(34.2 \%$ vs $65.8 \%$; $p=0.01)$

- were treated more prevalently with warfarin $(62.50 \%$ vs $37.50 \%$; $p=0.01$;
- had lower minimal nutritional assessment (MNA) total score $(24.26 \pm 2.69$ vs $25.92 \pm 2.99$ score; $\mathrm{p}=0.011)$;

- had a lower VAT level (14.25 \pm 4.65 vs $16.68 \pm 4.89$ score; $p=0.026) ;$ and

- had a lower left ventricular mass (228.18 $\pm 58.81 \mathrm{vs}$ $266.81 \pm 96.16 \mathrm{~g} ; \mathrm{p}=0.033$ ).

Moreover, AF patients with obesity (percentage FM for males $>25 \%$ and an for females $>35 \%$ ) compared to AF patients with lower percentage of FM, had, i.a. lower sVCAM-1 concentration indexed to percentage of FM (34.28 \pm 20.15 vs $48.30 \pm 32.45 ; p=0.020)$, lower LVM (232.01 \pm 69.45 vs $269.72 \pm 83.4 \mathrm{~g} ; \mathrm{p}=0.018)$, and lower LVM/BSA (LVMI; $116.84 \pm 31.17$ vs $131.03 \pm 37.43 ; p=0.045)$.

For both groups of patients, blood sVCAM-1 concentration correlated with the HAS-BLED score $(R=0.19$; $p=0.017)$, New York Heart Association (NYHA) class $(R=0.23 ; p=0.004), C C l(R=0.26 ; p=0.001)$, Nutritional Risk Screening 2002 (NRS-2002) score $(R=0.28$; 
$p<0.001)$, CRP $(R=0.20 ; p=0.013)$, NT-proBNP $(R=0.26 ; p<0.001)$; MNA score $(R=-0.34, p<0.001)$; and blood albumin concentration $(R=-0.26 ; p=0.001)$. Moreover, for both groups of patients, we identified only one statistically significant correlation: that between sVCAM-1 and LAD/BSA ( $R=0.19 ; p=0.02)$.

Among patients with $A F$, we found no significant correlations between SVCAM-1 and echocardiographic parameter values, or $\mathrm{CHA}_{2} \mathrm{DS}_{2} \mathrm{VASc}$ and HAS-BLED scores, which evaluates thromboembolic and hemorrhagic risk in patients with $A F$, respectively. However, these patients presented significant correlations between SVCAM-1 and parameters of BIA, such as FFM $(R=-0.23 ; p=0.037)$, SMM $(R=-0.22 ; p=0.048)$, and VAT score $(R=-0.27 ; p=0.014)$. In patients with $A F$, the relationships between SVCAM-1 and $\mathrm{CCl}$, NRS-2002, MNA, blood albumin and CRP concentrations were statistically significant and had similar power like that for both groups of patients (data not presented in detail).

\section{sVCAM-1 and the measured outcomes}

During, on average, $420.82 \pm 56.58$ days of follow-up, the prevalence of outcomes measured did not differ significantly between the two study groups (Tab. 1). Compared to patients who died during the follow-up ( $n=13,8.13 \%$ ), the individuals who survived for the whole of the observation period $(n=147)$ were statistically significantly younger, had higher body weight and nutritional scores (MNA, NRS-2002), lower blood NT-pro-BNP concentration, higher BIA parameters, lower LAD/BSA (detailed data not presented), and borderline lower serum SVCAM-1 concentration $(1228.66 \pm 598.23$ vs $1523.15 \pm 647.74 \mathrm{ng} / \mathrm{ml}$; $\mathrm{p}=0.093)$.

Eight of the 80 patients with AF (10.0\%) died during the observation period. These patients had only slightly higher serum sVCAM-1 concentration $(1397.86 \pm 721.88$ vs $1249.29 \pm 612.71 \mathrm{ng} / \mathrm{ml} ; \mathrm{p}=0.52)$ in comparison with AF patients who survived. Similar differences concerned patients with paroxysmal atrial fibrillation and arrhythmia recurrence, patients who required readmission or who had a stroke or myocardial infarction during the observation period (data not presented in detail).

For survival analysis, the cut-off value for sVCAM-1 was obtained in ROC analysis with the lowest Youden's index for the risk of all- cause mortality during follow-up (sVCAM-1 < and $\geq 1242.40 \mathrm{ng} / \mathrm{ml}$; the area under the curve (AUC) and 95\% confidence interval (Cl): 0.664; 0.507-0.0.820; $p=0.040$; Fig. 1 ), and for the risk of MACE during follow-up (sVCAM-1 < and $\geq 586.60 \mathrm{ng} / \mathrm{ml}$; AUC; 95\%Cl: 0.594; $0.501-0.687 ; p=0.049$ ). The Kaplan- Meier analysis showed that patients with higher SVCAM-1 had worse long term survival than their counterpart and the curves began to divide after 350 days after discharge (Fig. 2). The risk of all-cause mortality during follow-up for individuals with higher SVCAM-1 in whole patients group amounted to (OR; 95\%Cl): $5.39 ; 1.57-18.45$; $\mathrm{p}=0.007$. Of all studied patients and patients with $A F$, those who had higher SVCAM-1 concentration had also a higher risk of MACE (Fig. 3), with OR; $95 \% \mathrm{Cl}$ amounted to $6.00 ; 1.18-30.37 ; p=0.03$. We obtained also statistically significant logistic regression model for relationships between all- cause mortality during follow-up and blood sVCAM-1 concentration indexed to percentage of fat mass with $\mathrm{OR}, 95 \% \mathrm{Cl}$ per unit: 1.02 ; $1.00-1.04 ; p=0.019$.

\section{Discussion}

In this study, we tested the hypothesis of whether endothelial dysfunction, diagnosed on the basis of increased blood sVCAM-1 concentration, affects AF occurrence and patient prognosis (risk of all-cause mortality, readmission, or stroke). We did so because endothelial dysfunction is related to the risk of several cardiovascular diseases [3], to the presence of inflammation [12], to obesity and body composition $[9,13-19]$, as well as being involved in the risk of thromboembolic complications of AF [20-24]. We also investigated whether SVCAM-1 might link obesity with the pathogenesis of AF and patient prognosis $[10,11]$.

\section{sVCAM-1 and AF occurrence and patient prognosis}

In our study we confirmed the positive associations between abdominal obesity and risk of AF (Tab. 1) [10, 11], however, we did not corroborate the hypothesis that endothelial dysfunction, expressed by elevated sVCAM-1 is associated the risk of AF (Tab. 2), the risk of thromboembolic and hemorrhagic complications, expressed by $\mathrm{CHA}_{2} \mathrm{DS}_{2} \mathrm{VASc}$ and HAS-BLED scores. Therefore, our results did not corroborate the work summarized by Smiljic [3] and Wijesurendra and Casadei [4], who showed associations between an increased risk of AF and elevated levels of endothelial dysfunction biomarkers. Based on the 909 participants of the Bruneck Study, Willeit et al. [5] found higher blood sVCAM-1 concentration to be a predictor of new-onset AF in the general community. Moreover, in studies by Verdejo et al. [6] and Harling et al. [2], an increased blood sVCAM-1 concentration was a biomarker of AF occurrence after coronary artery bypass grafting (CABG). Associations between sVCAM-1 and AF occurrence were also reported by other authors [24-31]. In a study by Zhang et al. [32], blood sVCAM-1 concen- 


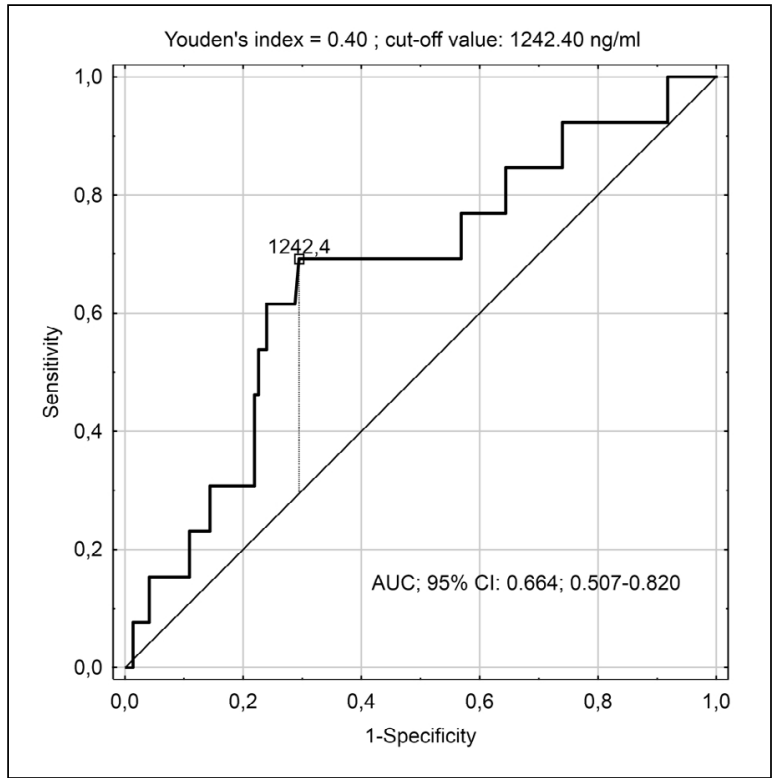

Figure 1. ROC curve for prediction of all-cause mortality by SVCAM-1 in both study groups

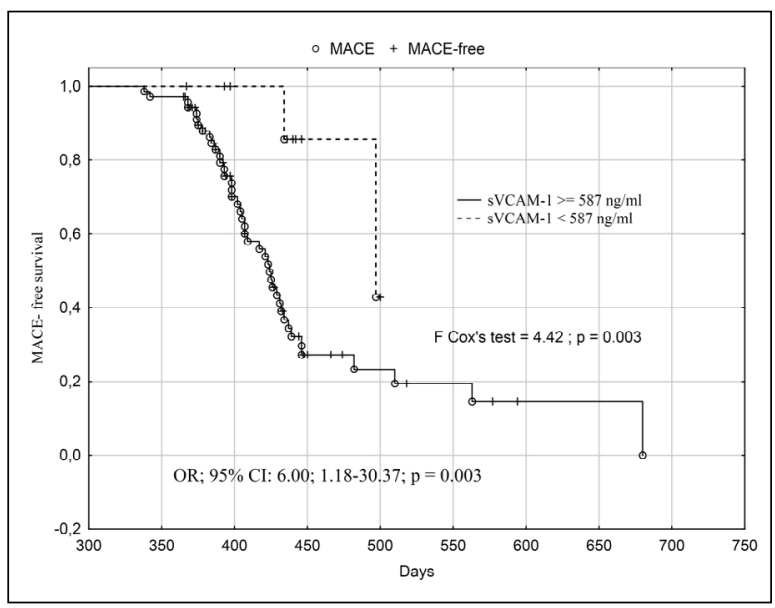

Figure 3. Risk of a major adverse cardiovascular event (MACE) in relation to sVCAM-1 concentration in KaplanMeier analysis for AF patients

tration was higher than in healthy controls, although this adhesion molecule lost its significance as a predictor of AF in multifactorial analysis. Seljeflot et al. [7] found impaired endothelial function, expressed by higher blood VWF and ADMA concentrations, in patients with AF who were over the age of 75 compared to an age-matched control group with sinus rhythm. However, similar to our results (Tab. 2), blood sVCAM-1 concentration was not higher to a statistically significant extent in the AF patients in their study.

Moreover, we found that elevated sVCAM-1 (Fig. 1, 2) and increase sVCAM-1 indexed to the percentage of FM (logistic regression) were associated with a sig-

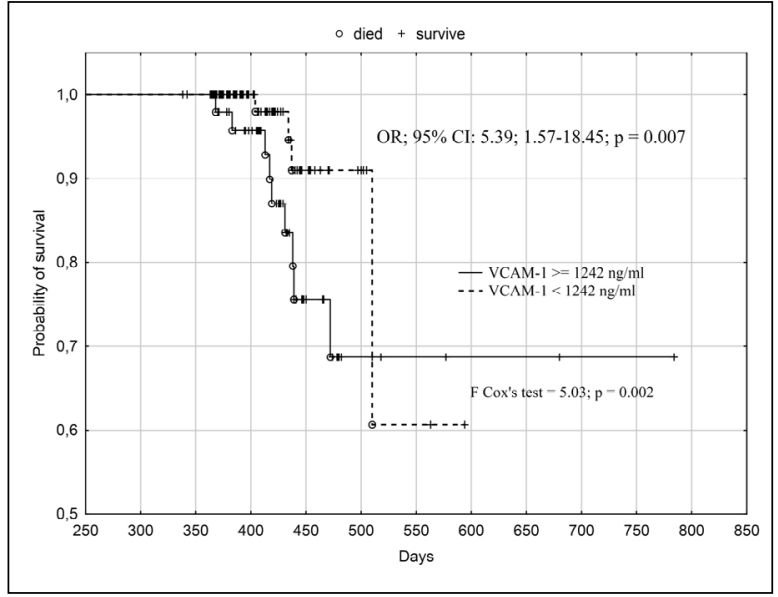

Figure 2. Risk of all-cause mortality in relation to sVCAM-1 concentration in Kaplan-Meier analysis for all study patients

nificant increase in the risk of all-cause mortality in the whole study group. Moreover, serum sVCAM-1 concentration $\geq 584 \mathrm{ng} / \mathrm{ml}$ linked higher MACE risk both in the whole study group, in AF patients group since 350 days after discharge (Fig. 3), and patients with exacerbation of chronic coronary syndromes. These data corroborate with reports showing on association between all-cause and cardiovascular mortality of patients with various cardiovascular disorders and endothelial dysfunction $[3,31]$ and elevated sVCAM-1 [5].

\section{sVCAM-1 and cardiovascular disease advancement and the risk of thromboembolic and hemorrhagic complications}

Breitenstein et al. [20] showed that, in isolated and cultured endocardial human cells of patients with $A F$, the endocardium of the left atrium had a higher expression of prothrombotic and pro-inflammatory markers, including sVCAM-1, than the endocardium of the right atrium. The role of blood prothrombotic state as a risk factor for thromboembolic complications in patients with AF was also described in analyses by Weymann et al. [31] and Polovina et al. [33]. However, Ruediger et al. [8] did not confirm the role of endothelial function as a marker for the difference in thromboembolic risk among Caucasian and Indian patients with AF. In our study, we found only weak positive Spearman correlations between sVCAM-1 and NYHA class, CCl score, blood NT-proBNP concentration, and left atrium size. Moreover, among patients with AF, we did not find significant correlations between SVCAM-1 and values of echocardiographic parameters or $\mathrm{CHA}_{2} \mathrm{DS}_{2} \mathrm{VASc}$. These discrepancies are difficult to explain.

However, we did find relationships between HASBLED score (for the whole study group) and the type of 
anticoagulant taken before the study (in the AF group). The HAS-BLED score correlated positively with blood sVCAM-1 concentration for the whole study group, and patients with AF whose blood SVCAM-1 concentration was above the median value took NOACs significantly less often than their counterparts. The first observation can be interpreted as an aggregate effect of different factors (e.g. hypertension, older age and renal insufficiency) inducing endothelial damage, and the second may be explained by the protective and reparative properties of rivaroxaban mediated by modification of urokinase plasminogen activator and matrix metallopeptidase-2 expression, as well as by the inhibiting pro-inflammatory effects of factor Xa [34, 35]. However, our patients took two different NOACs (dabigatran and rivaroxaban) and the small sample size made it impossible to analyze differences between the two drugs.

\section{sVCAM-1 and patient nutritional status and body composition}

The risk of AF showed a U-shaped relation to body mass [10]. One of the explanations for this relationship is the cardiac and vascular effect of adipocytokines [9, $10,11,13-17,19,30-37]$. It was reported that visfatin may induce expression of ICAM- 1 and VCAM- 1 and, in turn, leukocyte adhesion to endothelial cells and aortic endothelium [37]. In our study, blood sVCAM-1 concentration correlated negatively with the parameters of patient nutritional status (MNA and NRS-2002), and skeletal muscle mass (in BIA). Moreover, patients with AF had lower sVCAM-1 indexed to FM and VAT (Tab. 2), and AF patients with the increased percentage of FM had lower sVCAM-1, lower LVM and LVMI. Because of elevated SVCAM-1 in our study increased the risk of all-cause mortality by than five times and dysregulation in adipocytokines and endothelial injury markers are typical for obese patients $[19,36]$, this may suggest a protective effect of obesity on the risk of AF; part of what might be regarded as the "obesity paradox" [10]. The hypothesis is also supported by evidence of an increase in the percentage of fat mass reducing the value of the sVCAM-1-to-FM ratio, a rise that was slightly associated with the risk of all-cause mortality in logistic regression analysis.

\section{Strengths and limitations of the study}

The first potential strength of our study is its sample size, which was similar to or greater than those in the majority of the works cited [2, 6, 32]. Moreover, our study group consisted of consecutive, non-selected inpatients, which supports the assertion that our study was performed in a real-world population. The second strength of our study is the matching of the control group in relation to age and gender, which helps reduce the effect of a patient's age on systemic endothelial function. The main study limitation was that there were some differences in the clinical characteristics of the two groups studied (Tab. 1), particularly in relation to $\mathrm{CCl}$, which, due to the above-mentioned pathophysiological relations, might be an explanation for it not being possible to find a higher blood sVCAM-1 level in patients with AF compared to the control group (Tab. 2).

\section{Conclusions}

Elevated serum sVCAM-1 concentration was associated with the risk of all-cause mortality and MACE, but did not link the risk of AF and thromboembolic complications. The use of new oral anticoagulants in patients with AF may exert a favorable effect on endothelial function and be associated with lower SVCAM-1 serum concentration. A lower sVCAM-1 level in obese patients and decrease in risk of all-cause mortality with per unit increase of sVCAM-1 to FM ratio suggest that the "obesity paradox" that exists in patients with AF may be mediated by a lower level of endothelium damage markers.

Acknowledgement: This study was supported by a grant from Nicolaus Copernicus University for the basic scientific activity of the Department of Vascular and Internal Diseases.

\section{Declaration of conflict of interest: All the authors declare no conflict of interest.}

\section{References}

1. Nabi Aslan A, Baştuğ S, Ahmet Kasapkara H, et al. Coronary Artery Dominance May Predict Future Risk of Atrial Fibrillation. Acta Cardiol Sin. 2018; 34(4): 344-351, doi: 10.6515/ACS.201807_34(4).20180326B, indexed in Pubmed: 30065573.

2. Harling L, Lambert J, Ashrafian $\mathrm{H}$, et al. Pre-operative serum VCAM-1 as a biomarker of atrial fibrillation after coronary artery bypass grafting J Cardiothorac Surg. 2017; 12(1): 70, doi: 10.1186/s13019-017-0632-2, indexed in Pubmed: 28821262.

3. Smiljic S. The clinical significance of endocardial endothelial dysfunction. Medicina (Kaunas). 2017; 53(5): 295-302, doi: 10.1016/j. medici.2017.08.003, indexed in Pubmed: 29074341.

4. Wijesurendra RS, Casadei B. Atrial fibrillation: effects beyond the atrium? Cardiovasc Res. 2015; 105(3): 238-247, doi: 10.1093/cvr/cvv001, indexed in Pubmed: 25587048.

5. Willeit K, Pechlaner R, Willeit P, et al. Association Between Vascular Cell Adhesion Molecule 1 and Atrial Fibrillation. JAMA Cardiol. 2017; 2(5): 516-523, doi: 10.1001/jamacardio.2017.0064, indexed in Pubmed: 28355442

6. Verdejo H, Roldan J, Garcia L, et al. Systemic vascular cell adhesion molecule-1 predicts the occurrence of post-operative atrial fibrillation. Int J Cardiol. 2011; 150(3): 270-276, doi: 10.1016/j.ijcard.2010.04.033, indexed in Pubmed: 20447702.

7. Seljeflot I, R Ulimoen S, Enger S, et al. Asymmetric Dimethylarginine Levels are Highly Associated With Atrial Fibrillation in an Elderly Population. Cardiol Res. 2012; 3(3): 109-115, doi: 10.4021/cr175w, indexed in Pubmed: 28352406. 
8. Ruediger CD, John B, Kumar S, et al. Influence of ethnic background on left atrial markers of inflammation, endothelial function and tissue remodelling. Indian Pacing Electrophysiol J. 2018; 18(1): 1-5, doi 10.1016/j.ipej.2017.08.002, indexed in Pubmed: 29477215.

9. Spangenberg A, Maghsoodi N, Dulnoan D, et al. Bone Mineral Density and Body Composition are Associated with Circulating Angiogenic Factors in Post-menopausal Women. Calcif Tissue Int. 2016; 99(6) 608-615, doi: 10.1007/s00223-016-0186-7, indexed in Pubmed: 27572995

10. Anaszewicz M, Budzyński J. Clinical significance of nutritional status in patients with atrial fibrillation: An overview of current evidence. Cardiol. 2017; 69(5): 719-730, doi: 10.1016/j.jjcc.2016.06.014, indexed in Pubmed: 27520756.

11. Budzyński J, Anaszewicz M. The associations between atrial fibrillation and parameters of nutritional status assessment in the general hospital population - a cross-sectional analysis of medical documentation. Kardiol Pol. 2017; 75(3): 231-239, doi: 10.5603/KP.a2016.0182, indexed in Pubmed: 27995601

12. Li J, Solus J, Chen $Q$, et al. Role of inflammation and oxidative stress in atrial fibrillation. Heart Rhythm. 2010; 7(4): 438-444, doi: 10.1016/s. hrthm.2009.12.009, indexed in Pubmed: 20153266.

13. Adrielle Lima Vieira R, Nascimento de Freitas R, Volp AC. Adhesion molecules and chemokines; relation to anthropometric, body composition, biochemical and dietary variables. Nutr Hosp. 2014; 30(2): 223 236, doi: 10.3305/nh.2014.30.2.7416, indexed in Pubmed: 25208773

14. Chen YL, Chang CL, Sun CK, et al. Impact of obesity control on circulating level of endothelial progenitor cells and angiogenesis in response to ischemic stimulation. J Transl Med. 2012; 10: 86, doi 10.1186/1479-5876-10-86, indexed in Pubmed: 22568992.

15. Engin A. Endothelial Dysfunction in Obesity. Obesity and Lipotoxicity. 2017: 345-379, doi: 10.1007/978-3-319-48382-5 15.

16. Girerd N, Scridon A, Bessière F, et al. Periatrial epicardial fat is associated with markers of endothelial dysfunction in patients with atrial fibrillation. PLoS One. 2013; 8(10): e77167, doi: 10.1371/journal. pone.0077167, indexed in Pubmed: 24143210

17. Wycherley TP, Brinkworth GD, Keogh JB, et al. Long-term effects of weight loss with a very low carbohydrate and low fat diet on vascular function in overweight and obese patients. J Intern Med. 2010; 267(5) 452-461, doi: 10.1111/j.1365-2796.2009.02174.x, indexed in Pubmed: 20141567

18. Scridon A, Morel E, Nonin-Babary E, et al. Increased intracardiac vascular endothelial growth factor levels in patients with paroxysmal, but not persistent atrial fibrillation. Europace. 2012; 14(7): 948-953, doi: 10.1093/europace/eur418, indexed in Pubmed: 22308085.

19. Yu GIm, Jun SE, Shin DH. Associations of VCAM-1 gene polymorphisms with obesity and inflammation markers. Inflamm Res. 2017; 66(3): 217-225, doi: 10.1007/s00011-016-1006-2, indexed in Pubmed 27853845

20. Breitenstein A, Glanzmann M, Falk V, et al. Increased prothrombotic profile in the left atrial appendage of atrial fibrillation patients. Int J Cardiol. 2015; 185: 250-255, doi: 10.1016/j.ijcard.2015.03.092, indexed in Pubmed: 25814212.

21. Chung NAY, Belgore F, Li-Saw-Hee FL, et al. Is the hypercoagulable state in atrial fibrillation mediated by vascular endothelial growth factor? Stroke. 2002; 33(9): 2187-2191, doi: 10.1161/01. str.0000023889.84649.3d, indexed in Pubmed: 12215585.

22. Namba S, Yamaoka-Tojo M, Kakizaki R, et al. Erratum to: Effects on bone metabolism markers and arterial stiffness by switching to rivaroxaban from warfarin in patients with atrial fibrillation. Hear Vessels. 2017; 32(8): 983, doi: 10.1007/s00380-017-0966-7, indexed in Pubmed: 28314975
23. Freestone $B$, Chong AY, Lim HS, et al. Angiogenic factors in atrial fibrillation: a possible role in thrombogenesis? Ann Med. 2005; 37(5): 365-372, doi: 10.1080/07853890510037392, indexed in Pubmed: 16179272

24. Choudhury A, Freestone B, Patel J, et al. Relationship of soluble CD40 ligand to vascular endothelial growth factor, angiopoietins, and tissue factor in atrial fibrillation: a link among platelet activation, angiogenesis, and thrombosis? Chest. 2007; 132(6): 1913-1919, doi: 10.1378/chest.07-1565, indexed in Pubmed: 18079224.

25. Abe I, Teshima $\mathrm{Y}$, Kondo $\mathrm{H}$, et al. Association of fibrotic remodeling and cytokines/chemokines content in epicardial adipose tissue with atrial myocardial fibrosis in patients with atrial fibrillation. Heart Rhythm. 2018; 15(11): 1717-1727, doi: 10.1016/j.hrthm.2018.06.025, indexed in Pubmed: 29908372

26. Watson T, Shantsila E, Blann A, et al. Circulating progenitor cells in patients with atrial fibrillation and their relation with serum markers of inflammation and angiogenesis. Thromb Haemost. 2010; 104(2): 327-334 doi: 10.1160/TH10-01-0024, indexed in Pubmed: 20508899.

27. Tan $Q$, Zhang S, Qi X et al. Permanent atrial fibrillation impairs the function of circulating endothelial progenitor cells. Postgrad Med. 2017; 129(2): 198-204, doi: 10.1080/00325481.2017.1288063, indexed in Pubmed: 28128678

28. Peller M, Lodziński P, Ozierański K, et al. The influence of the atrial fibrillation episode duration on the endothelial function in patients treated with pulmonary veins isolation. Adv Clin Exp Med. 2017; 26(1): 109-113, doi: 10.17219/acem/66995, indexed in Pubmed: 28397441.

29. Ogi $\mathrm{H}$, Nakano $Y, N$ iida $S$, et al. Is structural remodeling of fibrillated atria the consequence of tissue hypoxia? Circ J. 2010; 74(9): 1815-1821, doi: 10.1253/circj.cj-09-0969, indexed in Pubmed: 20631454

30. Ferri $\mathrm{C}$, Desideri $\mathrm{G}$, Valenti $\mathrm{M}$, et al. Early upregulation of endothelial adhesion molecules in obese hypertensive men. Hypertension. 1999; 34(4 Pt 1): 568-573, doi: 10.1161/01.hyp.34.4.568, indexed in Pubmed: 10523328

31. Weymann A, Sabashnikov A, Ali-Hasan-Al-Saegh S, et al. Predictive Role of Coagulation, Fibrinolytic, and Endothelial Markers in Patients with Atrial Fibrillation, Stroke, and Thromboembolism: A Meta-Analysis, Meta-Regression, and Systematic Review. Med Sci Monit Basic Res. 2017: 23: 97-140, doi: 10.12659/MSMBR.902558, indexed in Pubmed: 28360407

32. Zhang Lu, Ding Ru, Zhen Yi, et al. Relation of urotensin II levels to Ione atrial fibrillation. Am J Cardiol. 2009; 104(12): 1704-1707, doi: 10.1016/j.amjcard.2009.07.053, indexed in Pubmed: 19962480.

33. Polovina MM, Lip GYH, Potpara TS. Endothelial (dys)function in lone atrial fibrillation. Curr Pharm Des. 2015; 21(5): 622-645, doi: 10.21 74/1381612820666140825143028, indexed in Pubmed: 25175088.

34. Álvarez E, Paradela-Dobarro B, Raposeiras-Roubín S, et al. Protective, repairing and fibrinolytic effects of rivaroxaban on vascular endothelium. Br J Clin Pharmacol. 2018; 84(2): 280-291, doi: 10.1111/bcp.13440, indexed in Pubmed: 28940408.

35. Kim JB, Joung HJ, Lee JM, et al. Evaluation of the vascular protective effects of new oral anticoagulants in high-risk patients with atrial fibrillation (PREFER-AF): study protocol for a randomized controlled trial. Trials. 2016; 17(1): 422, doi: 10.1186/s13063-016-1541-8, indexed in Pubmed: 27558002

36. Abd El-Kader SM, Al-Jiffri OH. Impact of weight reduction on insulin resistance, adhesive molecules and adipokines dysregulation among obese type 2 diabetic patients. Afr Health Sci. 2018; 18(4): 873-883, doi: 10.4314/ahs.v18i4.5, indexed in Pubmed: 30766550.

37. Kim SR, Bae YH, Bae SK, et al. Visfatin enhances ICAM-1 and VCAM-1 expression through ROS-dependent NF-kappaB activation in endothelial cells. Biochim Biophys Acta. 2008; 1783(5): 886-895, doi: 10.1016/j. bbamcr.2008.01.004, indexed in Pubmed: 18241674 